\title{
Komplikationsrate, Patientenzufriedenheit und klinischer Einsatz nach Implantation eines Port- kathetersystems mit Zulassung zur Hochdruckinfusion
}

\author{
Experiences with Power-Injectable Port Systems: Complications, Patient \\ Satisfaction and Clinical Benefit
}

Autoren

Institute
D.-H. Chang ${ }^{1}$, C. Kabbasch ${ }^{1}$, H. Bovenschulte ${ }^{1}$, M. Libicher ${ }^{2}$, D. Maintz ${ }^{1}$, C. Bangard ${ }^{1}$

Institut für Radiologische Diagnostik, Uniklinik Köln

2 Institut für diagn. Radiologie, Diakonie-Klinikum, Schwäbisch Hall
Key words

- angiography

- ultrasound

- percutaneous

- central venous catheter

power injection

eingereicht 7.5.2012

akzeptiert $\quad 21.12 .2012$

Bibliografie

Dol http://dx.doi.org/

10.1055/s-0032-1330713

Online-Publikation: 2013

Fortschr Röntgenstr 2013; 185:

454-460 @ Georg Thieme

Verlag KG Stuttgart · New York .

ISSN 1438-9029

Korrespondenzadresse Dr. De-Hua Chang

Institut für Radiologische Diagnostik, Uniklinik Köln

Kerpenerstr. 62

50924 Cologne

Germany

Tel.: ++49/176/62129034

Fax: ++49/32123211978

dchang@email.de

\section{Zusammenfassung}

$\nabla$

Ziel: Evaluation der Komplikationen, der Patientenzufriedenheit und des klinischen Einsatzes eines Portkathetersystems, welches zur Hochdruckinfusion zugelassen ist.

Material und Methoden: Es erfolgte eine Auswertung der seit 09/2010 - 05/2011 zur Portimplantation überwiesenen Patienten an einer universitären Einrichtung. Der Zugang erfolgte sonografisch gesteuert über die laterale V. subclavia. Technischer Erfolg der Implantation und akute Komplikationen wurden dem radiologischen Interventionsprotokoll entnommen. Langzeitkomplikationen, der klinische Einsatz des Portsystems und die Patientenzufriedenheit wurden sowohl aus Angaben im RIS/ KIS als auch nach 6 Monaten im Rahmen eines Telefongesprächs erhoben.

Ergebnisse: Es wurden insgesamt 195 Portkatheterimplantationen bei 193 Patienten (122 Frauen, 71 Männer, Durchschnittsalter: 48 Jahre, 15-81 Jahre) durchgeführt. Der technische Erfolg lag bei 99,5\%. Die durchschnittliche Katheterliegezeit betrug 169 Tage (insgesamt 29210 Katheterliegetage, Minimum: 14, Maximum: 251 Tage). Die Rate der Früh- und Spätkomplikationen betrug 0,82 pro 1000 Katheterliegetage $(n=24)$ bei 138 Patienten in der Verlaufskontrolle. 13/140 Portkathetersystemen $(9 \%)$ wurden aufgrund einer Komplikation vorzeitig explantiert. Die Patientenzufriedenheit mit dem Port war hoch (zufrieden/sehr zufrieden: 94\%). 22 von 138 Patienten (15,9\%) gaben postinterventionell subjektive Beschwerden an. Das Portkathetersystem wurde bei 34/209 (16\%) CT/MRT Untersuchungen zur i. v. KM-Gabe benutzt. Komplikationen während oder nach der KM-Gabe über den Port traten nicht auf.

Schlussfolgerung: Die Verwendung des PowerPort-Systems für KM-Injektionen im Rahmen von CT/MRT-Untersuchungen ist eine sichere Alternative zur oftmals schwierigen Punktion peripherer

\section{Abstract \\ $\nabla$}

Purpose: Evaluation of complications, patient satisfaction and clinical benefit of port systems with authorization for high pressure injection of contrast agent during $\mathrm{CT} / \mathrm{MR}$ examinations.

Materials and Methods: Ultrasound-guided insertions of central venous port catheters were performed through the lateral subclavian vein at a university teaching hospital. The radiological information system (HIS/RIS) was used to evaluate technical success and complication rates. Assessment of patient satisfaction and clinical benefit was carried out by a questionnaire during a telephone call 6 months after implantation of the port system.

Results: A total of 195 port systems in 193 patients were implanted. The catheter remained in place for a mean duration of 169 days (overall 29,210 catheter days). The technical success rate was $99.5 \%$ and the overall complication rate was $17.4 \%$ (24/138; 0.82 per 1000 catheter days). Follow-up revealed 13 early port explantations (9\%). Most of the patients reported high satisfaction in general (satisfied/very satisfied: 94\%). 34/209 contrast-enhanced CT/MRT scans (16\%) were performed using the port for contrast media injection. There were no complications during or after administration of contrast agent via the port system.

Conclusion: The Powerport system is a safe alternative for peripheral i.v. contrast media injection during CT/MR scans, but has been infrequently used. Most patients reported high overall satisfaction with the port system. 
Venen, wird bisher aber zu selten genutzt. Die Patientenzufriedenheit und Akzeptanz des Portsystems sind hoch.

\section{Einleitung}

Implantierbare Portkathetersysteme sind insbesondere bei onkologischen Patienten bei der intravenösen Applikation von Chemotherapeutika und parenteralen Nährstoffzufuhr von großer Bedeutung. Dauerhaft implantierte Portkatheter bieten einen sicheren, vielfach wieder verwendbaren Zugangsweg und ersparen den Patienten oftmals die schwierige Punktion peripherer Venen zur Applikation der Chemotherapie sowie die störenden Verschlüsse der oberflächlichen Armvenen. Aufgrund der Grunderkrankung sind bei Patienten mit langfristigem, zentralvenösem Zugang oftmals mehrfache computertomografische oder kernspintomografische Verlaufsuntersuchungen in kurzen Abständen zum Staging indiziert. Untersuchungstechniken in der Computertomografie (CT) und Magnetresonanztomografie (MRT) benötigen die rasche Injektion großer Kontrastmittelmengen, um eine zeitliche Trennung der verschiedenen Kontrastierungsphasen zu ermöglichen. Auf diese Weise wird eine differenzialdiagnostische Einteilung von Tumoren oder das Erkennen verschiedener Pathologien wie eine Lungenembolie in vielen Fällen erst möglich. Insbesondere in der CT und in der Angio-MRT gehören deshalb Hochdruckinjektionen zur Routine. Für diese Untersuchungsprotokolle wäre die Verwendung der zentralvenösen Zugänge im Grunde genommen ideal. Allerdings sind die meisten langfristig implantierten Zugänge nicht für die bei der Hochdruckinjektion erreichbaren Drücke (Flussgeschwindigkeit von bis zu $5 \mathrm{ml} / \mathrm{s}$ und einer Druckbegrenzung bis 300 psi) zugelassen [1]. Um Patienten mit schlechten peripheren Venenverhältnissen wiederholte und mehrmalige Venenpunktionen zu ersparen, werden seit September 2010 in unserem Institut spezielle Portkathetersysteme, die auch für die Hochdruckkontrastmittelinjektion in der CT und MRT geeignet sind, eingesetzt.

In der vorliegenden Arbeit soll die Patientenzufriedenheit mit dem neuen Portsystem und der klinische Einsatz der neuen Portkathetersysteme für die KM-Applikation bei CT- und MRT- Untersuchungen überprüft werden. Die Komplikationsraten bei der Implantation und beim späteren Gebrauch sollen im Vergleich zu einer vorangegangen retrospektiven Studie mit einem Standardportsystem [2] evaluiert werden.

\section{Material und Methoden}

Es erfolgte eine Auswertung der seit 09/2010 - 05/2011 zur Portimplantation überwiesenen Patienten an einer universitären Einrichtung. Insgesamt wurden in diesem Zeitraum bei 193 Patienten (122 Frauen, 71 Männer) 195 sonografisch gesteuerte Portkatheteranlagen durchgeführt. Der Zugang erfolgte in 138 Fällen über die linke, in 57 Fällen über die rechte V. subclavia. Das mittlere Lebensalter betrug 48 Jahre (Minimum: 15; Maximum: 81 Jahre). Bei 187 Patienten (97\%) erfolgte die Portimplantation vor geplanter Chemotherapie, bei 4 Patienten (2\%) zur Durchführung einer Langzeitantibiose, in 2 Fällen (1\%) zur parenteralen Ernährung. Die Portkatheteranlage erfolgte prinzipiell ambulant, sofern die Patienten nicht aus anderen medizinischen Gründen bereits stationär aufgenommen waren. Postinterventionell wurde allen Patienten ein Portausweis mit Produktbeschreibung ausgehändigt. Bei ambulanten Patienten wurden standardmäßig am Folgetag eine Wundkontrolle und Verbandswechsel in unserer Abteilung durchgeführt. Im weiteren Verlauf wurden die Patienten von den jeweiligen zuweisenden Ambulanzen des Klinikums weiter betreut. Portexplantationen wurden sowohl in unserem Institut als auch in der gynäkologischen und chirurgischen Klinik durchgeführt.

\section{Technik der Portimplantation}

Der Eingriff erfolgte in einer angiografischen Einrichtungseinheit in Lokalanästhesie unter sterilen Kautelen. Die Seite der Punktion richtete sich im Allgemeinen nach der Händigkeit des Patienten (bei Rechtshändern erfolgte die Implantation links und umgekehrt). Bei Patientinnen mit einem Mammakarzinom erfolgte die Portimplantation auf der kontralateralen Seite und bei Patienten mit einem Bronchialkarzinom auf der ipsilateralen Seite. Vor dem eigentlichen Eingriff erfolgte die farbdopplersonografische Darstellung der Durchgängigkeit der V. subclavia. Unter sterilen Kautelen wurde eine lokale Infiltrationsanästhesie des Zugangswegs durchgeführt und im Anschluss die V. subclavia im lateralen Abschnitt infraklavikulär sonografisch gesteuert punktiert (Sonografiegerät: Acuson X 300, Siemens Healthcare). Nach Einbringen der im Portset (Bard PowerPort isp M. R. I., Bard Access Systems, Inc, USA) enthaltenen Peel-off-Schleuse in Seldinger-Technik wurde der Katheter eingewechselt und unter Röntgendurchleuchtung in der V. cava superior vor dem rechten Vorhof platziert. Als Referenz einer optimalen Katheterlage während der Portanlage galt die Position der Spitze 2 Wirbelkörperhöhen unterhalb der Trachealbifurkation. Die Lage der Spitze $3 \mathrm{~cm}$ oberhalb oder unterhalb dieser gedachten Linie war innerhalb der Toleranz [3].

Nach erneuter Infiltrationsanästhesie erfolgte die stumpfe Präparation der Porttasche 2 Querfinger infraklavikulär und ca. $5 \mathrm{~cm}$ lateral des Sternums in der Fossa pectoralis. Der Katheter wurde subkutan von der Punktionsstelle bis zur Porttasche getunnelt, der Katheter auf die entsprechende Länge gekürzt, mit der Portkammer konnektiert und diese mit 2 Einzelknopfnähten an der Pektoralisfaszie fixiert. Nach erfolgreicher Aspiration von Blut wurde das System im Anschluss mit heparinisierter Kochsalzlösung (Heparin $250 \mathrm{IE} / \mathrm{ml}$ ) gespült. Der Wundverschluss erfolgte mit einer Subkutan- und Intrakutannaht. Die Intrakutannaht wurde nach 7 - 10 Tagen entfernt. Die Blutstillung erfolgte ausschließlich durch manuelle Kompression oder Umstechung ohne Einsatz eines Elektrokauters. Periinterventionell erfolgte eine Antibiotikaprophylaxe (Cephazolin Fresenius 2g; Fresenius Kabi, Bad Homburg).

\section{Komplikationsraten und Verlaufskontrollen}

Es erfolgte die retrospektive, deskriptiv statistische Auswertung der radiologischen Interventionsprotokolle sämtlicher Patienten, die zur Portimplantation überwiesen wurden. Es wurden erfasst: der technische Erfolg der Portimplantation, das Auftreten akuter Komplikationen, Datum der Portexplantation (falls erfolgt) und Grund für die Portexplantation. Das Interventionsprotokoll enthielt im Einzelnen Informationen über Zuweiser, Name und Alter des Patienten, rechtfertigende Indikation, Anamnese einschließlich Voruntersuchungen sowie ein ausführliches OP-Protokoll. Die in unserem Institut erfolgten radiologischen Nachfolgeuntersuchungen mittels kontrastmittelgestützter CT und MRT (CE-CT) 
MRT) wurden dem RIS (Radiologie-Informationssystem) entnommen und bezüglich Zeitpunkt, Indikation und aufgetretenen Komplikationen während der KM-Injektion ausgewertet. Die verwendeten Flussgeschwindigkeiten der KM-Injektion für CT- und MRT-Untersuchungen liegen in unserem Institut, in Abhängigkeit der Indikation, zwischen 0,5-4 ml/s. Laut Herstellerangaben soll unter Verwendung der im Portset enthaltenen Portnadel (20 Gauge) ein maximaler Druck bei KM-Infusion von 300 PSI (pounds per square inch) oder eine Flussrate von $5 \mathrm{ml} / \mathrm{s}$ nicht überschritten werden.

Langzeitkomplikationen, der klinische Einsatz des Portsystems und die Patientenzufriedenheit wurden nach 6 Monaten anhand eines strukturierten Erhebungsbogens im Rahmen einer telefonischen Kontaktierung der Patienten erfasst. Bezüglich der Auswertung der Patientenzufriedenheit wurde dieser Erhebungsbogen bereits in vorangegangenen Studien in leicht abgewandelter Form angewendet [4]. Mit einem Punkteschema von 1-4 wurden folgende Bewertungskriterien abgefragt: a) allgemeine Zufriedenheit mit dem Portsystem, b) kosmetisches Ergebnis, c) Empfehlung an Freunde/Bekannte, d) Benutzerfreundlichkeit. Im Falle a/b) 1=sehr zufrieden, 2=zufrieden, 3= unzufrieden, $4=$ sehr unzufrieden; Im Falle c) 1 =definitive Empfehlung, 2=eingeschränkte Empfehlung, 3=eher keine Empfehlung, 4 = definitiv keine Empfehlung; Im Falle d) 1 =sehr gut, 2 = gut, 3 = befriedigend, 4 = mangelhaft.

Es wurde weiter nach der Präferenz einer Portimplantation in Vollnarkose statt wie üblich in Lokalanästhesie gefragt. Falls eine subjektiv störende Belastung nach Portimplantation vorhanden war, sollte diese frei formuliert werden. In der Zwischenzeit aufgetretene Komplikationen und deren Therapie sowie auswärtig durchgeführte KM-gestützte CT/MRT-Untersuchungen wurden im Patientengespräch ermittelt und dokumentiert. Die Ursache für eine Nichtbenutzung des Portsystems für CE-CT/-MRT Untersuchungen aus Patientensicht wurde erfasst.

Die Komplikationen wurden nach den Empfehlungen der Society of Interventional Radiology (SIR) nach Schweregrad in Minorund Majorkomplikationen differenziert [5] sowie das zeitliche Auftreten in periinterventionelle/akute $(<24 \mathrm{~h})$, Früh- (bis zum 30.Tag) und Spätkomplikationen (ab dem 31.Tag) eingeteilt. Eine Minorkomplikation wurde definiert als Ereignis, welches keiner zusätzlichen operativen oder medikamentösen Therapie $>24 \mathrm{~h}$ bedurfte oder eine stationäre Aufnahme $<24 \mathrm{~h}$ zur Beobachtung. Eine Majorkomplikation beinhaltet jede Komplikation, die einer zusätzlichen operativen oder dauerhaften medikamentösen Therapie bedarf, einen stationären Aufenthalt $>24$ h oder Tod des Patienten.

Eine Portinfektion wurde diagnostiziert nach bakteriellem Kulturnachweis aus einem Abstrich aus der Porttasche oder am Portkatheter selbst. Bei Auftreten thrombosetypischer klinischer Symptome wurde eine duplexsonografische Untersuchung der porttragenden Extremität durchgeführt und bei nicht eindeutigem Befund durch eine KM-gestützte CT ergänzt.

\section{Ergebnisse}

$\nabla$

Insgesamt verstarben 33 von 193 Patienten $(17,1 \%)$ im Beobachtungszeitraum, 22 Patienten $(11,4 \%)$ wurden nicht in der Nachbeobachtung erfasst. Damit fallen 55/193 Patienten (28,5\%) aus der abschließenden Bewertung der Patientenzufriedenheit, da ein telefonisches Abschlussgespräch nicht durchführbar war. Bis zur letztmöglichen Dokumentation wurde bei 193 Patienten eine Katheterliegezeit von 29210 Tagen (Min.: 14, Max.: 251, Mittelwert: 169) erfasst. Die kurze durchschnittliche Verweildauer von unter einem halben Jahr (169 Tage) erklärt sich aus der Zusammensetzung unseres Patientenguts ( $\bullet$ Tab. 1$)$. Lediglich bei 4 Patienten ( $2 \%$ ) wurde die Portanlage für eine Langzeitantibiose und in 2 Fällen (1\%) zur parenteralen Ernährung durchgeführt. Die überwiegende Mehrzahl (97\%; 187/193) der Patienten erhielt eine Portimplantation zur Durchführung einer neo-/adjuvanten Chemotherapie.

\section{Technische Erfolgsrate}

In 194/195 Fällen (99,5\%) war die Portkatheteranlage erfolgreich. Ein Anlageversuch scheiterte aufgrund einer zentralen Thrombose. In 6 Fällen $(3,1 \%)$ gelang die Implantation nach primär erfolglosem Punktionsversuch der V. subclavia auf der Gegenseite. Ursache für die erschwerte Punktion war das Vorliegen eines sehr schmalkalibrigen Gefäßes.

Die Portimplantation erfolgte insgesamt durch 8 Radiologen, von denen 5 bereits mehr als 60 Portanlagen eigenständig durchgeführt hatten und 3 im Rahmen der Facharztweiterbildung neu eingearbeitet wurden.

\section{Periinterventionelle und akute Komplikationen ( $<24 \mathrm{~h}$ )}

Bei 4/195 Portkatheteranlagen (2,1\%) trat trotz Einsatz des Ultraschalls ein iatrogener Pneumothorax auf. 3 von 4 Komplikationen traten bei 2 Weiterbildungsassistenten in der Einarbeitungsphase (Erfahrung jeweils < 20 Portanlagen) auf. Hierbei war ein Pneumothorax drainagebedürftig (Majorkomplikation) und beinhaltete einen mehrtägigen stationären Aufenthalt. Bei 3 Patienten bildete sich der Pneumothorax im Verlauf spontan zurück. Diese Patienten wurden zur Beobachtung über eine Nacht stationär aufgenommen (Minorkomplikation).

\section{Frühkomplikationen (<30 Tage nach Intervention)}

Es traten 3 Majorkomplikationen bei 140 Patienten in der Frühphase auf $(2,1 \%)$.

Bei 2 Patienten (1,4\%) kam es zu einer postoperativen Infektion der Porttasche (4-9 Tage), die die Explantation des Portsystems notwendig machte. Bei einem Patienten wurde zu einem späteren Zeitpunkt eine erneute Portanlage durchgeführt. Wir verzeichneten eine Portdysfunktion ( $0,7 \%)$, die bedingt war durch eine Diskonnektion des Katheters vom Portreservoir.

\section{Spätkomplikationen (> 30 Tage nach Intervention)}

Es trat im weiteren Verlauf bei 21/138 Patienten (15,2\%) eine Spätkomplikation (31 - 526 Tage nach Portimplantation) auf. Darunter fallen 18 Major- (13,0\%) und 3 Minorkomplikationen $(2,2 \%)$.

\begin{tabular}{|c|c|}
\hline Mammakarzinom & 69 \\
\hline NHL & 25 \\
\hline Bronchialkarzinom & 15 \\
\hline Morbus Hodgkin & 11 \\
\hline Ovarialkarzinom & 10 \\
\hline Rhabdomyosarkom/Chondrosarkom & 9 \\
\hline Malignes Melanom & 8 \\
\hline Plattenepithelkarzinom & 8 \\
\hline Ösophaguskarzinom & 7 \\
\hline sonstige & 31 \\
\hline
\end{tabular}




\begin{tabular}{|c|c|c|c|}
\hline & $\begin{array}{l}\text { Anzahl } \\
\text { (n) }\end{array}$ & $\begin{array}{l}\text { Zeitintervall nach } \\
\text { Implantation (Tage) }\end{array}$ & Therapie \\
\hline \multicolumn{4}{|l|}{ Minorkomplikation } \\
\hline Pneumothorax & 3 & 0 & spontane Remission \\
\hline Umschlagen des Katheters & 3 & Median: 87 (Range: 78 - 98) & $\begin{array}{l}2 \times \text { interventionelle Repo- } \\
\text { sitionierung des Katheters }\end{array}$ \\
\hline gesamt & 6 & & \\
\hline \multicolumn{4}{|l|}{ Majorkomplikation } \\
\hline $\begin{array}{l}\text { Portlagerinfektion oder systemische } \\
\text { Infektionen im Rahmen einer kathe- } \\
\text { terassoziierten Bakteriämie }\end{array}$ & 9 & Median: 50 (Range: 4 - 113) & $9 \times$ Portexplantation \\
\hline Venenthrombose & 11 & Median: 55 (Range: 28 - 132) & $\begin{array}{l}11 \times \text { systemische } \\
\text { Antikoagulation }\end{array}$ \\
\hline Pneumothorax & 1 & 0 & Drainageanlage \\
\hline Katheterdiskonnektion & 1 & 22 & $\begin{array}{l}\text { Katheterbergung und } \\
\text { Portexplantation }\end{array}$ \\
\hline gesamt & 22 & & \\
\hline
\end{tabular}

Tab. 2 Aufteilung der Komplikationen nach Schweregrad (Minor-/ Majorkomplikationen).
Es erfolgte bei 10 Patienten (7,3\%) die vorzeitige Portexplantation aufgrund einer vermuteten Infektion der Porttasche oder im Rahmen einer systemischen Infektion auf dem Boden einer katheterassoziierten Bakteriämie. In 3 Fällen konnte kein Keimnachweis von der Katheterspitze gewonnen werden, sodass die Ursache einer systemischen Infektion unklar blieb. Definitionsgemäß traten demnach 7 Portinfektionen (5,1\%) in der Spätphase auf. Es wurde bei 11 Patienten (8,0\%) eine Thrombose der porttragenden Armvene per FKDS oder Computertomografie (CT) diagnostiziert. Davon fielen 7 Patienten klinisch durch eine Armschwellung auf. In 4 Fällen wurde als Zufallsbefund, im Rahmen des onkologischen Stagings, computertomografisch eine Thrombose nachgewiesen. Eine Patientin ging in der Nachbeobachtung verloren. Die übrigen Patienten erhielten eine systemische Antikoagulation bis zur vollständigen Ausheilung oder bis zur Portkatheterentfernung nach Abschluss der Chemotherapie.

Bei 3 Patientinnen $(2,1 \%)$ kam es zu einem Umschlagen des Portkatheters in der ipsilateralen/kontralateralen V. brachiocephalica. Eine Patientin war adipös (BMI: 33), 2 Patientinnen normalgewichtig (BMI: 20 - 22). In 2 Fällen konnte der Katheter interventionell unter Durchleuchtung mit einer Fassschlinge wieder in eine korrekte Lage überführt werden. Bei einer Patientin wurde nach Abschluss der Therapie eine elektive Portexplantation vorgenommen.

\section{Zusammenfassung der Früh- und Spätkomplikationen}

Die Rate der Früh- und Spätkomplikationen betrug 0,82 pro 1000 Katheterliegetage (24/138 Patienten; 17,4\%) im Nachbeobachtungszeitraum. Hierunter fielen 3 Minor- und 21 Majorkomplikationen. Eine Übersicht der Minor- und Majorkomplikationen unter Berücksichtigung des zeitlichen Auftretens gibt $\bullet$ Tab. 2 wieder.

Es erfolgten 43 Portexplantationen, davon 17 in unserem Institut, 16 in der gynäkologischen Klinik, 9 in der chirurgischen Klinik und eine in einer auswärtigen Klinik. 13/140 Portkathetersystemen (9\%) wurden aufgrund einer Komplikation vorzeitig explantiert. Ursache war eine Portkatheterdiskonnektion und im übrigen Infektionen des Portsystems (12/140, wobei bei 3 der explantierten Portkatheter kein Keimnachweis gelang). Häufigste Früh- und Spätkomplikation ist die Thrombose der porttragenden Vene (11/138; $8 \%)$. Es fand sich keine signifikante Korrelation der Komplikationshäufigkeit oder der Schwere einer Kom-
Tab.3 Ergebnisse des Erhebungsbogens bezüglich der Patientenzufriedenheit $(n=138)$.

\begin{tabular}{|c|c|c|c|c|}
\hline Frage & 1 & 2 & 3 & 4 \\
\hline allgemeine Zufriedenheit ${ }^{1}$ & 48 & 82 & 7 & 1 \\
\hline kosmetisches Ergebnis $^{1}$ & 30 & 89 & 18 & 1 \\
\hline Empfehlung an Freunde ${ }^{2}$ & 124 & 10 & 4 & 0 \\
\hline Benutzerfreundlichkeit ${ }^{3}$ & 93 & 42 & 2 & 1 \\
\hline
\end{tabular}

plikation in Bezug auf das Geschlecht oder die zugrundeliegende Diagnose.

Es bleibt zu erwähnen, dass vollständige Informationen zum postinterventionellen Verlauf nur von 138/193 Patienten (71,5\%) vorliegen. 55/193 Patienten waren nach 6 Monaten nicht mehr erreichbar. Komplikationen, die bei diesen Patienten auftraten, wurden aus dem RIS- und dem KIS-Patientensystem erfasst und in Bezug auf die gesamte Katheterliegezeit von 193 Patienten bis zur letzten schriftlichen Dokumentation berechnet. Es ist daher eher von einer etwas höheren tatsächlichen Komplikationsrate auszugehen.

\section{Patientenzufriedenheit mit dem Portsystem}

Insgesamt 138/193 Patienten (71,2\%) nahmen an der Befragung mittels standardisiertem Erhebungsbogen nach 6 Monaten teil.

Die Gesamtzufriedenheit mit dem Portsystem war hoch. Eine Zusammenfassung der Ergebnisse gibt $\bullet$ Tab. 3 wieder. Wird die Gruppe der Patienten zusammengefasst, die mit dem Portsystem zufrieden oder sehr zufrieden ist, so ergibt sie einen Anteil von 130/138 (94,2\%). 8 Patienten (5,8\%) waren mit dem Portsystem allgemein unzufrieden oder sehr unzufrieden. Grund für die Unzufriedenheit war in 5 Fällen das Auftreten einer katheterassoziierten Komplikation, in 2 Fällen ein subjektiv störender, weit über dem Hautniveau abstehender Port und in einem Fall ein schwer anzustechender Port bei Adipositas per magna (BMI: 40). Mit dem kosmetischen Ergebnis war die überwiegende Mehrheit zufrieden oder sehr zufrieden (119/138, 86,2\%). 18 Patienten waren unzufrieden (13,0\%), nur eine sehr unzufrieden (0,7\%). Ursache der Unzufriedenheit war ein subjektiv weit über dem Haut- 
niveau abstehender Port ( $\mathrm{n}=13$; 11 Frauen, 2 Männer) und/oder eine starke Keloidbildung/Hautverfärbung ( $n=6$, 6 Frauen).

124 Patienten (90,0\%) würden, falls notwendig, ihren Freunden oder Bekannten ein Portsystem definitiv weiterempfehlen. 10 Patienten würden sich nur eingeschränkt und 4 Patienten eher keine Empfehlung für das Portsystem aussprechen. Als Grund für eine eingeschränkte/keine Empfehlung gaben die Patienten an: Port stört, weil weit vom Hautniveau abstehend $(n=3)$, Keloidbildung $(n=1)$, Schmerzen bei der Implantation $(n=3)$, im Verlauf aufgetretene Komplikation $(n=3)$, keine spezifische Angabe einer Ursache $(n=4)$.

Bezüglich der Benutzerfreundlichkeit erreicht das Portsystem in der überwiegenden Zahl eine positive Resonanz. 93 Patienten (67,4\%) schätzen die Benutzerfreundlichkeit als sehr gut, 42 Patienten $(30,0 \%)$ als gut, 2 Patienten (1,5\%) als befriedigend und einer $(0,7 \%)$ als mangelhaft ein.

25 von 138 Patienten $(18,1 \%)$ würden sich retrospektiv für eine Vollnarkose entscheiden. Als Ursache wurden Schmerzen während des Eingriffs 17/25 (68\%), die Dauer des Eingriffs 6/25 (24\%) und Angst oder der Eingriff per se 2/25 (8\%) angegeben. Für die übrigen Patienten war der Eingriff in Infiltrationsanästhesie gut tolerabel.

22 von 138 Patienten (15,9\%) bejahten die Frage nach einer subjektiv unangenehmen oder störenden Belastung in den letzten 6 Monaten nach der Portimplantation. So empfanden 4 der $22 \mathrm{~Pa}-$ tienten die unmittelbar postinterventionellen Schmerzen als unangenehm. Für weitere 5 Patienten war die im Verlauf aufgetretene Komplikation vor allem psychisch belastend. Jeweils 5 der Patienten hatten Probleme beim Schlafen und Liegen auf der Seite des Ports oder beim Autofahren aufgrund eines Druckgefühls durch den Anschnallgurt. Für 2 der Patientinnen war das kosmetische Ergebnis aufgrund des abstehenden Portsystems die größte subjektive Belastung. 1 Patient klagte über eine geringe Bewegungseinschränkung.

\section{Verwendung des Portsystems für kontrastmittelge- stützte CT- und MRT(CE-CT/-MRT)-Untersuchungen} 209 KM-gestützte CT- und MRT-Untersuchungen wurden bei 91 Patienten durchgeführt. Im Durchschnitt erhielt jeder dieser Patienten 2,3 Untersuchungen (Minimum: 1 Untersuchung; Maximum: 8 Untersuchungen). Hiervon wurden 180/209 Untersuchungen innerhalb unseres Instituts und 29/209 Untersuchungen an externen radiologischen Einrichtungen durchgeführt. In 34 von 209 Fällen (16,3\%) wurde das Portsystem für die i.v. KMInjektion verwendet. Hierbei traten keine Komplikationen auf. Die Häufigkeit der CE-CT/-MRT-Untersuchungen im Vergleich zur Verwendung des Portsystems zur KM Injektion veranschaulicht Abb. 1. Tab. 4 fasst die Ursachen für die Nichtnutzung des Portsystems aus Patientensicht zusammen. Sowohl am eigenen Institut als auch an externen Häusern wurde vor allem die Unsicherheit beim Personal bezüglich der Zulassung des Portsystems oder im Umgang mit dem Port selbst als Grund für eine Nichtverwendung angegeben. In 102 von 209 Fällen (48,8\%) einer CT/MRT-Untersuchung wurde daher trotz Patientenwunsch die Portbenutzung durch das Personal abgelehnt. In 73 von 209 Fällen $(34,9 \%)$ wurde von Seiten des Patienten kein Wunsch zur Portnutzung geäußert.

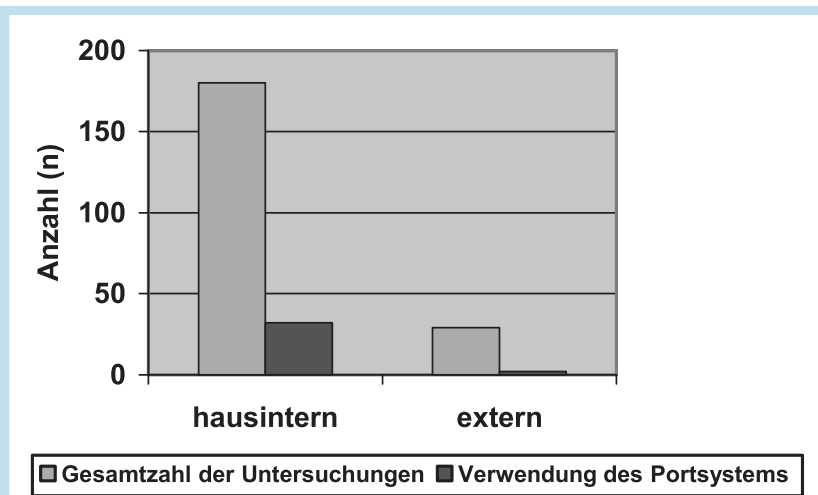

Abb. 1 Vergleich der Gesamtzahl der CE-CT/MRT-Untersuchungen und der Häufigkeit der Verwendung des Portsystems für die Kontrastmittelinjektion.

Tab. 4 Grund für die Nichtverwendung des Portsystems bei CE-CT/MRTUntersuchungen aus Patientensicht $(n=175)$.

\begin{tabular}{lcc}
\hline Ursache & intern & extern \\
\hline $\begin{array}{l}\text { Unsicherheit beim Personal/Ablehnung durch } \\
\text { Personal }\end{array}$ & 85 & 13 \\
\hline $\begin{array}{l}\text { Benutzung des Ports durch Patient nicht explizit } \\
\text { gewünscht }\end{array}$ & 62 & 11 \\
\hline keine Portnadel vorhanden & 1 & 3 \\
\hline gesamt & 148 & 27 \\
\hline
\end{tabular}

\section{Diskussion \\ $\nabla$}

Der Zugangsweg über die V. jugularis wurde 2003 in unserem Institut verlassen. Seitdem werden Standardportsysteme sonografisch gesteuert über die V. subclavia implantiert. Ursache hierfür war eine regelhafte Demarkierung des subkutanen Katheterverlaufs über der Klavikula, der insbesondere bei schlanken und kachektischen Patienten als kosmetisch störend empfunden wurde. Damit sind wir auf ein Akzeptanzproblem sowohl auf Seiten der Patienten als auch der zuweisenden Kollegen gestoßen. Der Wechsel des Portkatheters auf ein System, welches für Hochdruckinfusionen zugelassen ist, erfolgte 09/ 2010 zeitgleich mit dem Beginn der vorliegenden Studie unter Beibehaltung der Implantationstechnik. Der Vergleich mit einer vorangegangenen retrospektiven Studie, welche die Erfolgsund Komplikationsraten der Implantation des Standardportsystems (Fresenius Intraport II Keramik) bei 1517 Patienten/1532 Portkatheteranlagen über einen Zeitraum von 4 Jahren beschreibt [2], zeigt aktuell unverändert die hervorragende technische Erfolgsrate von 99,5\% (gegenüber 99,0\%). Weiterhin ist trotz Einsatz des Ultraschalls die Ausbildung eines Pneumothorax die häufigste, periinterventionell aufgetretene Komplikation $(4 / 195 ; 2,1 \%$ versus $22 / 1537 ; 1,4 \%)$.

Die Gesamtzahl der Früh- und Spätkomplikationen der vorliegenden Arbeit (24/138; 17,4\%, 0,82 pro 1000 Katheterliegetage) liegt im Bereich anderer Studien für Standard- und Hochdruckinfusionsportsysteme mit ähnlicher Patientenzahl [1, 6-9]. Die differenzierte Betrachtung der Komplikationen zeigt eine auffällig hohe Zahl an intravasal umgeschlagenen Kathetern in der Anfangszeit der Umstellung auf das neue Portsystem (3/138, 2,1\%). Die Komplikation trat untersucherunabhängig ausschließlich bei 

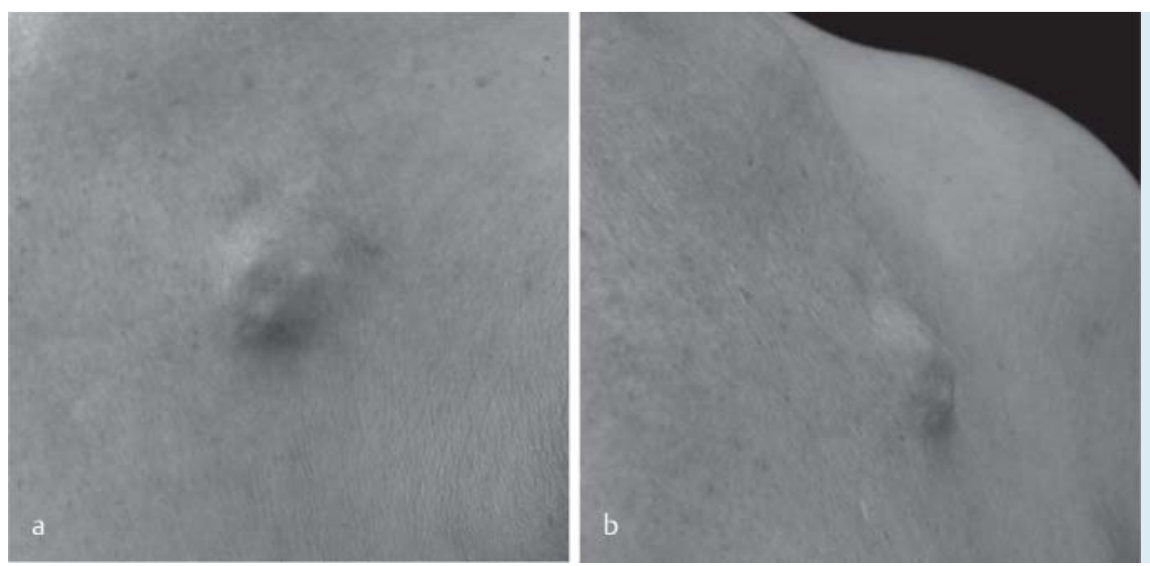

Abb. 2 a Frontansicht des implantierten Portreservoirs: Demarkierung mit deutlichem Abstehen vom Hautniveau. b Schräg seitliche Ansicht des implantierten Portreservoirs: deutliches Abstehen des Reservoirs vom Hautniveau.

weiblichen Patientinnen auf, wobei in allen Fällen die postinterventionelle Röntgenkontrolle eine Positionierung der Katheterspitze in der oberen Vena cava superior, etwa $3-5 \mathrm{~cm}$ oberhalb der tolerierten Grenze und $6-8 \mathrm{~cm}$ oberhalb der intendierten Lage ( 2 Wirbelkörperhöhen unterhalb der Trachealbifurkation) zeigte. Von einigen Autoren wird eine postinterventionell aufgetretene, zusätzliche Retraktion des Katheters einer Migration des Portreservoirs, vor allem bei Frauen mit voluminösen Brüsten, durch Lagewechsel aus dem Liegen in die stehende Position zugeschrieben $[1,10]$. In diesem Zusammenhang stellen Unterarmports möglicherweise eine bessere Alternative dar [11]. Allerdings trat dieses Problem zuvor beim Standardportsystem nicht auf. Neben einem systematischen Fehler spielen daher möglicherweise auch Materialeigenschaften des Katheters eine Rolle. Katheter beim Hochdruckportsystem bestehen aus Polyurethan. Sie besitzen aufgrund der höheren Knickstabilität geringere Wandstärken gegenüber den Silikonkathetern beim Standardportsystem [12, 13]. Es ist möglich, dass die Reduktion des Durchmessers des Katheters zu einer instabileren Lage des Katheters intravasal führt. Hier scheint die Lernkurve beim Umgang mit dem neuen Material eine Rolle zu spielen. In keinem Fall eines umgeschlagenen Portkatheters war zuvor eine CE-CT/-MRTUntersuchung erfolgt, sodass sich hier kein kausaler Zusammenhang ergibt. Weder ein Umschlagen des Katheters noch die in der Anfangszeit aufgetretene Diskonnektion aufgrund einer mangelhaften Befestigung des Katheters am Portreservoir traten in der Folgezeit auf. Bei einer Katheterlage im herznahen Abschnitt der V. cava superior und Fixierung des Portreservoirs nahe/auf der Pectoralisfaszie sollte das Risiko einer Retraktion und damit eines Umschlagens des Katheters minimiert werden.

Bei 2 Patienten (1,4\%) kam es trotz durchgeführter Single-ShotAntibiose am 4. und 9. postoperativen Tag zu einer Infektion der Porttasche, die die Explantation des Portsystems notwendig machte. In der Literatur wird die perinterventionelle Infektionsprophylaxe mittels Antibiotikagabe kontrovers diskutiert. Die Datenlage ist derzeit nicht eindeutig. Sowohl für als auch gegen eine periinterventionelle Antibiotikaapplikation gibt es in der Literatur gute Argumente aus randomisierten Studien [14-18]. Die thrombotische Komplikationsrate in unserer Patientengruppe lag mit 0,37 pro 1000 Katheterliegetage $(n=11)$ etwas höher als in bisher publizierten Studien, die zwischen 0,1-0,35 pro $1000 \mathrm{~d}$ für Subklaviaports angegeben wird [9, 19-22]. Allerdings wird in den meisten Studien die Thrombosehäufigkeit rein retrospektiv aus den vorhandenen Krankenakten ausgewertet, sodass von einer gewissen Dunkelziffer auszugehen ist und die tatsächliche Zahl der Thrombosen eher unterschätzt wird. Zukünftige
Studien sind notwendig, um den Wert neuerer Entwicklungen, wie beispielsweise imprägnierter oder beschichteter Katheter zur Thrombose-/Infektionsprophylaxe, abschätzen zu können [23-26].

Die Lebensqualität und Patientenzufriedenheit spielt bei langfristigen zentralvenösen Kathetern eine herausragende Rolle. Hier sind bisher wenige vergleichende Studien bezüglich der optimalen Katheterwahl und der Patientenzufriedenheit veröffentlicht. In unserer Studie wird eine hohe Patientenzufriedenheit mit dem Portkathetersystem erreicht. 130/138 Patienten (94,2\%) waren zufrieden oder sehr zufrieden, 124/138 Patienten (90,0\%) würden das Portsystem definitiv weiterempfehlen.

In der Subgruppenanalyse fällt auf, dass die Zahl der unzufriedenen Patienten vor allem bei Frauen auf das kosmetische Ergebnis zurückzuführen ist (19/138, 13,8\%, 17 Frauen; 2 Männer). Ursache war in den meisten Fällen $(n=13)$ eine zunehmende Demarkierung des subkutan gelegenen Portreservoirs. Insbesondere bei schlanken und kachektischen Patientinnen wurde das weit über das Hautniveau abstehende Portreservoir als kosmetisch störend empfunden. Nachteilig wirkt sich hier möglicherweise die etwas höhere Bauweise des Portreservoirs (Höhe: 15,3 mm) sowie die oberflächliche 3-Punkt- Markierung der Portmembran aus ( $\bullet$ Abb. 2a, b). Diese wiederum soll ein besseres Auffinden der Einstichstelle vor allem bei adipösen Patienten ermöglichen. Auf der anderen Seite sind diese Systeme für Patienten, bei denen im Verlauf ein Verlust von subkutanem Fettgewebe zu erwarten ist und damit die Gefahr einer Drucknekrose steigt, z. B. bei Portanlagen zur parenteralen Ernährung, eher nicht gut geeignet. Mittlerweile sind vom Hersteller zusätzlich flachere Low-ProfileSysteme auf dem Markt. Insbesondere Patienten mit wenig subkutanem Fettgewebe sollen hiervon profitieren.

25 von 138 Patienten (18,1\%) würden sich retrospektiv, wenn die Möglichkeit bestünde, für eine Vollnarkose entscheiden. 8 der Patienten (32\%) gaben die Länge der Prozedur oder Angst als Ursache für das Missempfinden an. Besondere Aufmerksamkeit sollte daher schwerkranken Patienten, die eine längere Prozedur in Rückenlage nicht tolerieren oder besonders ängstlichen Patienten geschenkt werden. Hier sollte großzügig und frühzeitig der Entschluss zur Behandlung in Narkose fallen.

Im Verlauf wurde das Portsystem in 34 Fällen zur i.v. KM-Gabe im Rahmen einer CT- oder MRT-Untersuchung verwendet. Es traten unter Begrenzung der Flussrate auf $\leq 4 \mathrm{ml} / \mathrm{s}$ keine Komplikationen im Rahmen der Injektionen auf. In anderen Studien konnte gezeigt werden, dass die Kontrastierung der Gefäße in der CT, gemessen in der Aorta, äquivalent zur CT-Untersuchung mit KM-Applikation über großlumige periphere Zugänge ist [27]. 
Frühere In-vitro-Studien versuchten bereits eine Rechtfertigung für die Verwendung von Standardportsystemen zur KM-Injektion aufzuzeigen. So konnte bei Verwendung einer geeigneten Druckbegrenzung von 325 PSI eine KM-Injektion von $2-4 \mathrm{ml} / \mathrm{s}$ ebenfalls sicher über ein Standardportkathetersystem appliziert werden, ohne dass eine Extravasation von KM festgestellt werden konnte [1]. Allerdings merken die Autoren kritisch an, dass die Food and Drug Administration 2004 eine Warnung bezüglich der Verwendung von zentralvenösen Zugängen bei der Hochdruckinjektion veröffentlicht hat [28]. Diese Warnung bezieht sich auf alle Typen zentralvenöser Katheter. Trotz der nun vorliegenden Zulassung zur Hochdruckinfusion über das neue Portsystem seitens des Herstellers zeigt sich in unserer Studie eine nur geringe Anwendung für derartige Untersuchungen. Das Portsystem wurde durchschnittlich lediglich bei jeder 6. CE-CT/-MRT-Untersuchung verwendet (34/209; 16,2\%). In 73 von 209 Fällen (34,9\%) wurde von Seiten des Patienten kein Wunsch zur Portnutzung geäußert, sodass oftmals dem Personal die Existenz eines Ports nicht bewusst war. Allerdings überwiegt die Zahl der Fälle (102/209; 48,8\%), bei denen trotz Patientenwunsch die Portbenutzung durch das Personal abgelehnt wurde. Die Unsicherheit beim Personal bezüglich der Verwendbarkeit des Portsystems und die häufige Notwendigkeit des Anstechens der Portkammer spielten eine Rolle für die geringe Nutzung. Von Herstellerseite wurde der Port mit einer röntgendichten Markierung mit der Aufschrift „CT“ versehen, der zusätzlich eine Zuordnung möglich macht. Entscheidend scheint jedoch eine konsequente Einarbeitung der Mitarbeiter in der Verwendung der Portsysteme (Anstechen, Spülen) zu sein, um den sicheren und reibungslosen Umgang zu gewährleisten.

Unsere Arbeit bestätigt eine exzellente technische Erfolgsrate bei der Implantation des neuen Hochdruckportsystems. Die Umstellung auf ein neues Portsystem kann durch eine Lernkurve im Umgang mit neuen Materialien zu einer höheren Frühkomplikationsrate führen. Die Benutzung für Kontrastmittelinjektionen bei CT/MRT-Untersuchungen ist sicher und kann empfohlen werden, wenn das radiologische Personal ausreichend im Umgang geschult wurde.

\section{Literatur}

1 Gebauer B, Teichgräber UK, Hothan T et al. Kontrastmitteldruckinjektion in Portkathetersysteme - Ergebnisse einer In-vitro-Studie. Fortschr Röntgenstr 2005; 177: 1417-1423

2 Chang DH, Boecker J, Hellmich $M$ et al. Ergebnisse sonographisch gesteuerter Portkatheterimplantationen über die laterale Vena subclavia: eine retrospektive Analyse bei 1532 Patienten. Fortschr Röntgenstr 22.05 2012, (Epub ahead of print)

3 Teichgräber UK, Nagel SN, Kausche S et al. Clinical benefit of power-injectable port systems: A prospective observational study. Eur J Radiol 2012; 81: 528-533

4 Quinn RR, Lamping DL, Lok CE et al. The vascular access questionnaire: assessing patient-reported views of vascular access. J Vasc Access 2008; 9: 122 - 128

5 Silberzweig JE, Sacks D, Khorsandi AS et al. Reporting standards for central venous access. J Vasc Interv Radiol 2000; 11: 391 - 400

6 Caers J, Fontaine C, Vinh-Hung $V$ et al. Catheter tip position as a risk factor for thrombosis associated with the use of subcutaneous infusion ports. Support Care Cancer 2005; 13: 325-331
7 Vandoni RE, Guerra A, Sanna P et al. Randomised comparison of complications from three different permanent central venous access systems. Swiss MedWkly 2009; 139: 313-316

8 Sticca RP, Dewing BD, Harris JD. Outcomes of surgical and radiologic placed implantable central venous access ports. Am J Surg 2009; 198 : 829-833

9 Vardy J, Engelhardt K, Cox K et al. Long-term outcome of radiologicalguided insertion of implanted central venous access port devices (CVAPD) for the delivery of chemotherapy in cancer patients: institutional experience and review of the literature. Br J Cancer 2004; 91: $1045-1049$

10 Inoue Y, Miyazaki N, Shimizu Y et al. Indwelling catheter retraction because of pendulous breast. JPEN J Parenter Enteral Nutr 2000; 24: $251-253$

11 Goltz JP, Machann W, Noack C et al. Feasibility of power contrast injections and bolus triggering during CT scans in oncologic patients with totally implantable venous access ports of the forearm. Acta Radiol 2011; 52: $41-47$

12 Cohen $A B$, Dagli M, Stavropoulos SW et al. Silicone and polyurethane tunneled infusion catheters: a comparison of durability and breakage rates. J Vasc Interv Radiol 2011; 22: 638-641

13 Lersch C, Paschalidis $M$, Theiss $W$. Tiefe Venenthrombosen durch zentralvenöse Katheter. Vasa 1999; 28: $71-78$

14 Gebauer B, Teichgräber $U$, Werk $M$ et al. Periinterventionelle prophylaktische Antibiotikagabe bei der radiologischen Portkatheterimplantation. Fortschr Röntgenstr 2007; 8: 804-810

15 McKee $R$, Dunsmuir $R$, Whitby $M$ et al. Does antibiotic prophylaxis at the time of catheter insertion reduce the incidence of catheter-related sepsis in intravenous nutrition? J Hosp Infect 1985; 6: 419-425

16 Ranson MR, Oppenheim BA, Jackson A et al. Double-blind placebo controlled study of vancomycin prophylaxis for central venous catheter insertion in cancer patients. J Hosp Infect 1990; 15: 95-102

17 Bock SN, Lee RE, Fisher $B$ et al. A prospective randomized trial evaluating prophylactic antibiotics to prevent triple-lumen catheter-related sepsis in patients treated with immunotherapy. J Clin Oncol 1990; 8: $161-169$

18 Al-Sibai MB, Harder EJ, Faskin RW et al. The value of prophylactic antibiotics during the insertion of long-term indwelling silastic right atrial catheters in cancer patients. Cancer 1987; 60: 1891 -1895

19 De Gregorio MA, Miguelena JM, Fernández JA et al. Subcutaneous ports in the radiology suite: an effective and safe procedure for care in cancer patients. Eur Radiol 1996; 6: 748 -752

20 Lorch $H$, Zwaan M, Kagel C et al. Central venous access ports placed by interventional radiologists: experience with 125 consecutive patients. Cardiovasc Radiol 2001; 24: 180-184

21 Morris SL, Jaques PF, Mauro MA. Radiology-assisted placement of implantable subcutaneous infusion ports for long-term venous access. Radiology 1992; 184: 149-151

22 Cadman A, Lawrance JA, Fitzsimmons L et al. To clot or not to clot? That is the question in central venous catheters. Clin Radiol 2004; 59: $349-$ 355

23 Gilbert R, Howard R, Mok Q. Heparin-bonded lines reduce hospitalacquired bacteraemia. J Hosp Infect 2003; 54: 163-164

24 Long DA, Coulthard MG. Effect of heparin-bonded central venous catheters on the incidence of catheter-related thrombosis and infection in children and adults. Anaesth Intensive Care 2006; 34: 481 - 484

25 Gastmeier P, Zuschneid I, Geffers C. Antimicrobially impregnated catheters: An overview of randomized controlled trials. J Vasc Access 2003; 4: $102-110$

26 Falagas ME, Fragoulis K, Bliziotis IA et al. Rifampicin-impregnated central venous catheters: a meta-analysis of randomized controlled trials. J Antimicrob Chemother 2007; 59: 359-369

27 Wieners G, Redlich $U$, Dudeck $O$ et al. First experiences with intravenous port systems authorized for high pressure injection of contrast agent in multiphasic computed tomography. Fortschr Röntgenstr 2009; 181: $664-668$

28 FDA. Reminders from FDA Regarding Ruptured Vascular Access Devices from Power Injection. 2004, http://www.fda.gov/cdrh/medicaldevice safety/tipsarticles/reminder-rvad.html 